Genome Insight

Genetics of Microorganisms

\title{
Genomic sequencing of different sequevars of Ralstonia solanacearum belonging to the Moko ecotype
}

\author{
Ana Karolina Leite Pais ${ }^{1}$ (D), Jessica Rodrigues da Silva ${ }^{2}$, Leandro Victor Silva dos Santos ${ }^{1}$, \\ Greecy Mirian Rodrigues Albuquerque ${ }^{1}$, Antonio Roberto Gomes de Farias ${ }^{1}$ (D), Wilson José Silva Junior ${ }^{3}$, \\ Valdir de Queiroz Balbino ${ }^{3}$, Adriano Márcio Freire Silva ${ }^{4}$, Marco Aurélio Siqueira da Gama ${ }^{1}$ and Elineide \\ Barbosa de Souza ${ }^{5}$ (D) \\ ${ }^{1}$ Universidade Federal Rural de Pernambuco (UFRPE), Departamento de Agronomia, Recife, PE, Brazil. \\ ${ }^{2}$ Instituto Federal de Educação, Ciência e Tecnologia do Piauí (IFPI), Piauí, PI, Brazil. \\ ${ }^{3}$ Universidade Federal de Pernambuco (UFPE), Departamento de Genética, Recife, PE, Brazil. \\ ${ }^{4}$ Universidade Federal de Alagoas (UFAL), Departamento de Agronomia, Maceió, AL, Brazil. \\ ${ }^{5}$ Universidade Federal Rural de Pernambuco (UFRPE), Departamento de Biologia, Recife, PE, Brazil.
}

\begin{abstract}
Banana vascular wilt or Moko is a disease caused by Ralstonia solanacearum. This study aimed to sequence, assemble, annotate, and compare the genomes of $R$. solanacearum Moko ecotypes of different sequevar strains from Brazil. Average nucleotide identity analyses demonstrated a high correlation (> 96\%) between the genome sequences of strains CCRMRs277 (sequevar IIA-24), CCRMRs287 (IIB-4), CCRMRs304 (IIA-24), and CCRMRsB7 (IIB-25), which were grouped into phylotypes IIA and IIB. The number of coding sequences present in chromosomes and megaplasmids varied from 3,070 to 3,521 and 1,669 to 1,750 , respectively. Pangenome analysis identified 3,378 clusters in the chromosomes, of which 2,604 were shared by all four analyzed genomes and 2,580 were single copies. In megaplasmids, 1,834 clusters were identified, of which 1,005 were shared by all four genomes and 992 were identified as single copies. Strains CCRMRsB7 and CCRMRs287 differed from the others by having unique clusters in both their chromosomes and megaplasmids, and CCRMRsB7 possessed the largest genome among all Moko ecotype strains sequenced to date. Therefore, the genomic information obtained in this study provides a theoretical basis for the identification, characterization, and phylogenetic analysis of $R$. solanacearum Moko ecotypes.
\end{abstract}

Keywords: Plant pathogenic bacteria, banana tree, NGS, bacterial wilt, Musa spp.

Received: June 02, 2020; Accepted: November 25, 2020.

Ralstonia solanacearum is a soil-inhabiting plant pathogenic bacterium that is known to infect several economically important crops (Wicker et al., 2007), including banana (Musa spp.). Upon infecting banana trees, $R$. solanacearum causes vascular wilt, also known as Moko disease. $R$. solanacearum species complex were subdivided into a hierarchical classification system (including phylotypes) based on sequence analysis of the 16S-23S internal transcribed spacer (ITS) region, the endoglucanase (egl) gene, and the $m u t S$ (DNA repair) genes (Prior and Fegan, 2005). Each phylotype was associated with to a geographic origin: phylotype I (Asia), phylotype II (the Americas), phylotype III (Africa), and phylotype IV (Indonesia; Genin and Denny, 2012). Moreover, strains were grouped into sequevars that exhibited variations of $\leq 1 \%$ within egl gene sequences (Fegan and Prior, 2005).

Nevertheless, since $2014, R$. solanacearum species complex have been reclassified into three different species according to their phylotypes: $R$. pseudosolanacearum (phylotypes I and III), R. solanacearum (phylotype II - IIA and IIB), and R. syzygi (phylotype IV and closely related

Send correspondence to Ana Karolina Leite Pais. Universidade Federal Rural de Pernambuco (UFRPE), Departamento de Agronomia, Av. D. Manoel de Medeiros, s/n Dois Irmãos, 52171-900, Recife, PE, Brazil. E-mail: karolinapais@gmail.com strains) (Safni et al., 2014). Among these, the strains that cause Moko disease belong to sequevars IIA-6, IIA-24, IIA41, IIA-53, IIB-3, IIB-4, and IIB-25 (Cellier and Prior, 2010; Albuquerque et al., 2014; Santiago et al., 2017).

There are currently 203 publicly-available $R$. solanacearum genome sequences deposited in the National Center for Biotechnology Information (NCBI) database, of which only 17 belong to sequevar isolates IIA-6, IIA-24, IIA-53, IIB-3, and IIB-4 of the Moko ecotype. In Brazil, $R$. solanacearum strains belonging to the Moko ecotype are considered quarantine pests restricted to the northern (Amazonas, Amapá, Pará, Rondônia, and Roraima) and northeastern (Alagoas and Sergipe) states (MAPA, 2018). Although Brazil is likely the biodiversity center of $R$. solanacearum (Santiago et al., 2017), there are only three genome sequences of Brazilian strains (IBSBF1900 - IIA24, IBSBF2570 - IIA-53, and SFC - IIA-53) deposited in the NCBI database. Therefore, our study sought to sequence, assemble, annotate, and compare the genomes of Brazilian $R$. solanacearum strains belonging to sequevars IIA-24, IIB-4, and IIB-25 of the Moko ecotype.

In this study, CCRMRs277 (IIA-24), CCRMRs287 (IIB-4), CCRMRs304 (IIA-24), and CCRMRsB7 (syn. B7; IIB-25) were isolated from Brazilian Amazon banana trees exhibiting Moko disease symptoms. Bacterial strains were cultivated on 
2,3,5-triphenyl tetrazolium chloride medium for $48 \mathrm{~h}$ at $28{ }^{\circ} \mathrm{C}$ and white-colored colonies with pink centers were selected for DNA extraction, which was performed using the PureLink ${ }^{\circledR}$ Genomic DNA Kit (Thermo Fisher Scientific, Waltham, MA, USA) following the manufacturer's instructions.

For genome sequencing, pair-end DNA library preparation was performed using the Illumina Nextera DNA Flex Prep Kit (Illumina, San Diego, CA, USA) following the manufacturer's recommendations, and sequencing was performed on an Illumina MiSeq-2500 sequencer (100 cycles). Read quality was first assessed with the FastQC software (Andrews, 2010), and sequence trimming was performed using Sickle v.1.33 (Joshi and Fass, 2011). All reads that met the quality control requirements were assembled de novo using Unicycler version 3 (Wick et al., 2017) and were then evaluated with Quast v.5.0.2 (Gurevich et al., 2013) to estimate genome size, contig number, N50, and GC content (\%). Additionally, single-copy orthologs were identified and gene content conservation was analyzed with the BUSCO software (Seppey et al., 2019).

The average nucleotide identity (ANI) between assembled genome sequences was obtained via global alignment with the MUMmer algorithm (Kurtz et al., 2004) using the Pyani 0.2.7 Python3 module (Pritchard et al., 2016). The ABACAS v.1.3.1 software (Assefa et al., 2009) was implemented with the PROmer and NUCmer algorithms (Kurtz et al., 2004) to perform chromosome and megaplasmid alignments using the Po82 and UW163 strains ( $R$. solanacearum Moko ecotype, sequevar IIB-4) as a reference, as these are the only strains whose whole genomes are deposited in the NCBI database. The QUAST software v.5.0.2 (Gurevich et al., 2013) was used to evaluate contigs and select alignment scaffolds with the lowest number of Ns and the largest number of predicted genes. Synteny and visualization of the four $R$. solanacearum genome alignments and the two reference genomes were performed using the Mauve software (Darling et al., 2004) and CGView Server (Grant and Stothard, 2008), respectively.

Genome annotation was performed using the RAST online platform (Brettin et al., 2015), which also groups genes into subsystems based on the reconstruction of metabolic systems. The pangenome was built based on data obtained from RAST annotation using the Orthovenn online platform (Wang et al., 2015) to identify clusters of specific genes/ orthologs for each strain.

The sequences of the four $R$. solanacearum genomes were assembled into different sizes, of which the CCRMRs287 genome was the smallest $(5,464,210 \mathrm{bp})$ and the CCRMRsB7 genome was the largest $(5,847,640 \mathrm{bp})$ (Table 1$)$. Compared to other Moko ecotype genome sequences available in the NCBI database, strain CCRMRsB7 was found to possess the largest genome sequence identified to date. The coverage between the four genome sequences varied from 129.8x (CCRMRs304) to 163x (CCRMRsB7). Additionally, different strains exhibited considerable variations in assembled contig number and N50 values; however, a gene conservation rate of $>97 \%$ was observed in all examined strains, indicating that the assemblies were reliable. Additional genome assembly details are summarized in Table 1.

ANIm analysis demonstrated a $>96 \%$ sequence similarity between the genome sequences, confirming that they belonged to the same species based on an ANIm cut-off value of 9596\% for species delineation (Richter and Rossello-Mora, 2009). Even though the genomes of all strains exhibited high sequence similarity, it was possible to differentiate the strains corresponding to phylotypes IIA (99.9\%) and IIB (98.7\%). Moreover, even though strain CCRMRsB7 (sequevar IIB-25) belongs to phylotype IIB, it formed a subdivision within this group (Figure 1). Nonetheless, this strain displayed ANIm values of $98 \%$ and $96.4 \%$ when compared with the other members of phylotypes IIB and IIA, respectively.

Table 1 - Characteristics of the genome sequences of four different $R$. solanacearum strains of the Moko ecotype from Brazil.

\begin{tabular}{|c|c|c|c|c|c|c|c|c|}
\hline Features & \multicolumn{2}{|c|}{ CCRMRs277 } & \multicolumn{2}{|c|}{ CCRMRs $287^{\mathrm{b}}$} & \multicolumn{2}{|c|}{ CCRMRs304 ${ }^{c}$} & \multicolumn{2}{|c|}{ CCRMRsB $^{\mathrm{d}}$} \\
\hline Coverage & \multicolumn{2}{|c|}{133} & \multicolumn{2}{|c|}{148} & \multicolumn{2}{|c|}{129.8} & \multicolumn{2}{|c|}{163} \\
\hline Size before alignment (bp) & \multicolumn{2}{|c|}{5.659 .851} & \multicolumn{2}{|c|}{5.464 .210} & \multicolumn{2}{|c|}{5.654 .054} & \multicolumn{2}{|c|}{5.847 .640} \\
\hline GC content $(\%)$ & \multicolumn{2}{|c|}{66.54} & \multicolumn{2}{|c|}{66.59} & \multicolumn{2}{|c|}{66.55} & \multicolumn{2}{|c|}{66.46} \\
\hline Number of contigs & \multicolumn{2}{|c|}{360} & \multicolumn{2}{|c|}{92} & \multicolumn{2}{|c|}{368} & \multicolumn{2}{|c|}{88} \\
\hline N50 & \multicolumn{2}{|c|}{43.891} & \multicolumn{2}{|c|}{150.675} & \multicolumn{2}{|c|}{45.590} & \multicolumn{2}{|c|}{216.010} \\
\hline BUSCO (\%) & \multicolumn{2}{|c|}{97.97} & \multicolumn{2}{|c|}{98.65} & \multicolumn{2}{|c|}{97.97} & \multicolumn{2}{|c|}{97.97} \\
\hline \multirow[t]{2}{*}{ Size after alignment (bp) } & \multicolumn{2}{|c|}{5.636 .326} & \multicolumn{2}{|c|}{5.444 .697} & \multicolumn{2}{|c|}{5.645 .239} & \multicolumn{2}{|c|}{5.854 .658} \\
\hline & Chrom $^{\mathrm{e}}$ & Plasmid $^{\mathrm{f}}$ & Chrom & Plasmid & Chrom & Plasmid & Chrom & Plasmid \\
\hline References strains & $\mathrm{PO}^{2} 2^{g}$ & UW163 ${ }^{\mathrm{h}}$ & UW163 & UW163 ${ }^{\text {h }}$ & $\mathrm{PO}^{2} 2^{\mathrm{g}}$ & UW163 ${ }^{\text {h }}$ & $\mathrm{PO}^{2} 2^{\mathrm{h}}$ & UW163 \\
\hline Size after alignment (bp) & 3.549 .795 & 2.086 .531 & 3.512 .030 & 1.932 .667 & 3.549 .663 & 2.095 .576 & 3.716 .474 & 2.138 .184 \\
\hline Coding sequences & 3.099 & 1.718 & 3.315 & 1.669 & 3.070 & 1.720 & 3.521 & 1.750 \\
\hline Subsytems number & 299 & 81 & 318 & 83 & 291 & 88 & 321 & 93 \\
\hline RNAs & 46 & 1 & 52 & 1 & 45 & 1 & 51 & 4 \\
\hline
\end{tabular}

${ }^{\mathrm{a}-}$ CCRMRs277 = sequevar IIA-24; ${ }^{\text {b- }}$ CCRMRs287 = sequevar IIB-4; ${ }^{\mathrm{c}-}$ CCRMRs304 = sequevar IIA-24; ${ }^{\mathrm{d}-}$ CCRMRsB7 = sequevar IIB-25.

e- Chromosome;

f- Megaplasmid;

g. NUCmer alignment algorithm;

h-PROmer alignment algorithm. 


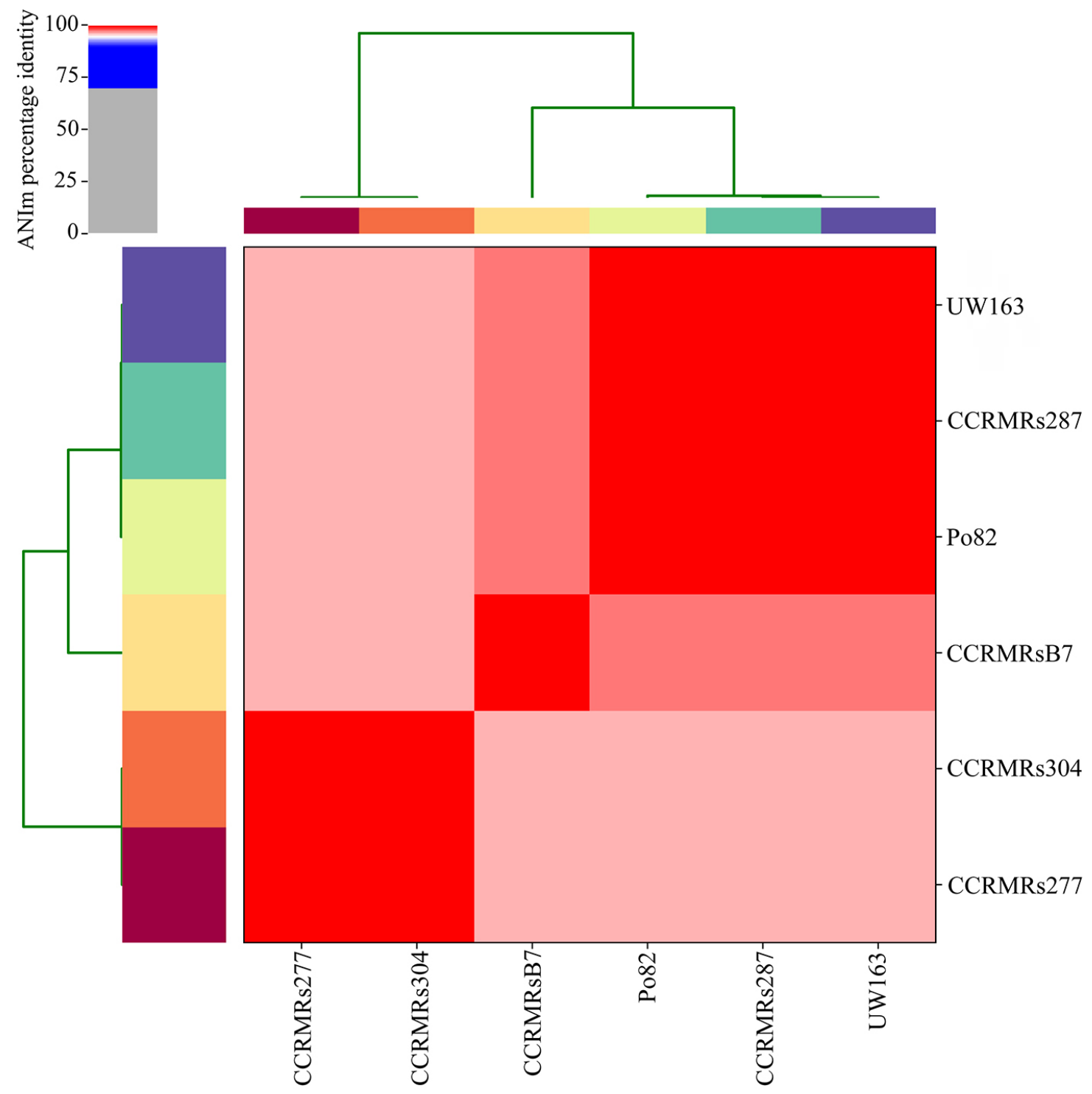

Figure 1 - Average Nucleotide Identity (ANIm) of four R. solanacearum strains belonging to the Moko ecotype (CCRMRs277, sequevar IIA-24; CCRMRs287, sequevar IIB-4; CCRMRs304, sequevar IIA-24; and CCRMRsB7, sequevar IIB-25) from Brazil, as well as the employed reference strains (UW163 and Po82, sequevar IIB-4).

The $R$. solanacearum complex possesses chromosomes and megaplasmids, and our study determined the lowest number of Ns and the largest number of predicted genes in the replicons. The chromosome scaffolds of CCRMRs277, CCRMRs304, and CCRMRsB7 were formed from alignments with Po82, and the chromosome scaffold of CCRMRs287 and all megaplasmid scaffolds had UW163 as a reference (Table 1).

The synteny of the genomic sequences is illustrated in Figure 2A and 2B, for Po82 and UW163, respectively. In both cases, the linear arrangement of the genomes exhibited a high level of collinearity for the two replicons. However, we also observed translocations and inversions of the locally collinear blocks (LCBs). When Po82 was used as a reference (Figure 2A), inversions were detected in the blue- and violet-colored LCBs and the violet-colored LCB of the CCRMRs287 and CCRMRsB7 strains, respectively. Similarly, when UW163 was used as a reference (Figure 2B), we noted that compared with the other three strains, there were substantial rearrangements in the pink- and green-colored LCBs of the CCRMRs287 strain. We assume that these features are attributable to the evolutionary relatedness of the CCRMRs287 and UW163 (sequevar IIB-4) strains, as well as the proximity of their geographical origins (the cities of Benjamin Constant-BR, and Nauta-PE, respectively, on the border between Brazil and Peru). Although this process has yet to be clarified, it is known to be a common event and can lead to adaptive phenotypic effects, as in the case of transposable elements that carry genes related to antibiotic resistance (Ceccatto, 2015). The circular visualization of the alignment of the genomic sequences produced by CGView showed that most of the genomic regions were highly conserved between the genomes (Figure 2C and 2D). 

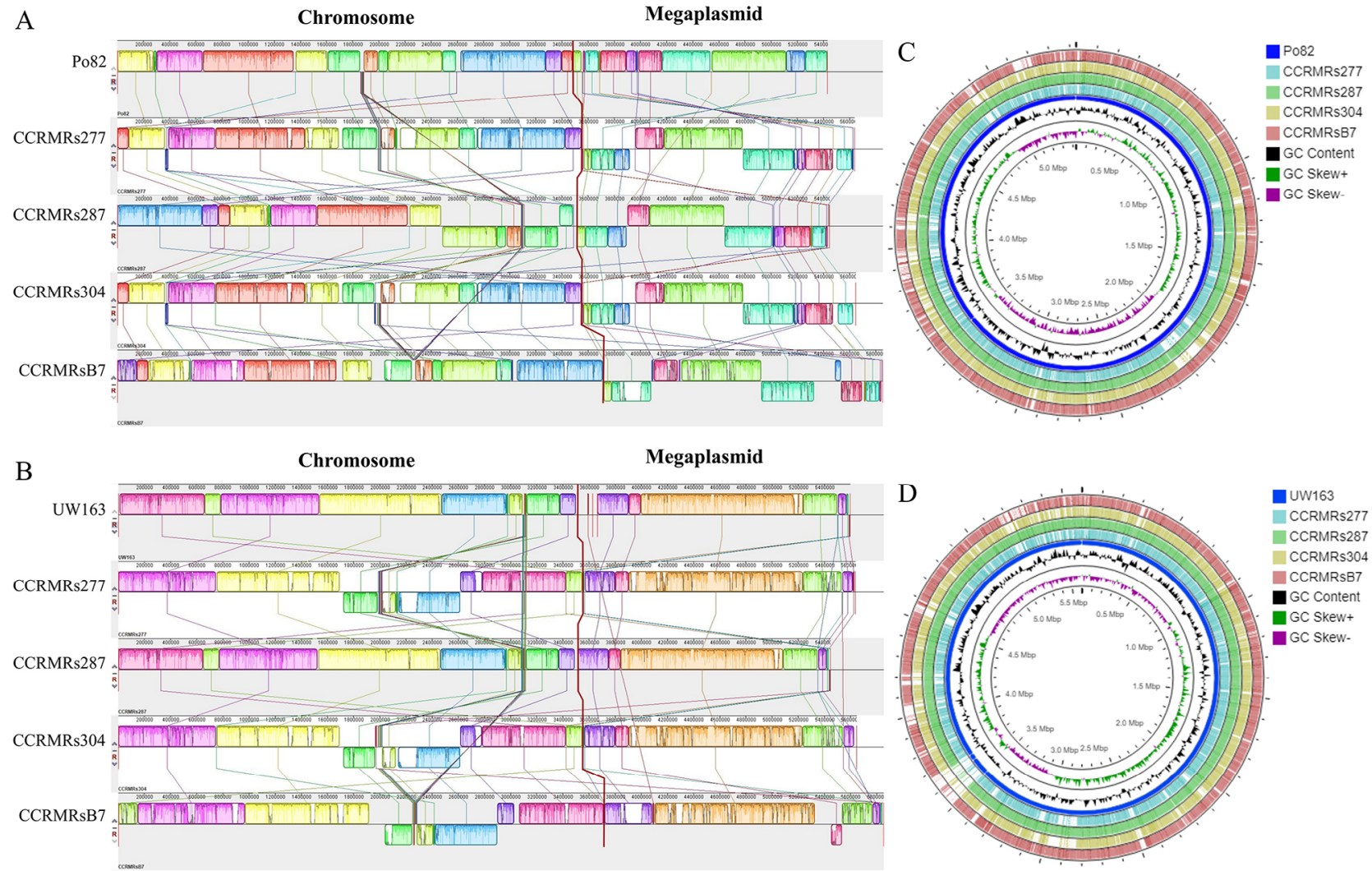

Figure 2 - Synteny of four $R$. solanacearum strains belonging to the Moko ecotype (CCRMRs277, sequevar IIA-24; CCRMRs287, sequevar IIB-4; CCRMRs304, sequevar IIA-24; and CCRMRsB7, sequevar IIB-25) from Brazil and reference strains Po82 (A) and UW163 (B). Visualization of the chromosome and megaplasmid alignments of the $R$. solanacearum strains and reference strains Po82 (C) and UW163 (D).

Table 1 details the number of coding sequences (CDS), RNAs, and subsystems of the genome assemblies of strains CCRMRs277, CCRMRs287, CCRMRs304, and CCRMRsB7, as well as the reference genomes and algorithms used for assembly. The chromosomes from the four genomes exhibited higher CDS, RNAs, and subsystem numbers, and therefore contained more information than megaplasmids. This observation may be related to the size and conservation of the chromosome, which represents the larger region (Genin and Denny, 2012) and is more conserved than the megaplasmid (Guidot et al., 2007).

The four most represented subsystems found in the four $R$. solanacearum chromosomes were (I) amino acids and derivatives; (II) protein metabolism; (III) carbohydrates; and (IV) cofactors, vitamins, prosthetic groups, and pigments, which accounted for $49-51 \%$ of the total subsystems (Figure 3A). For the megaplasmids, only $15 \%$ of the annotated genes were grouped into subsystems of which the most represented were (I) membrane transport; (II) virulence, disease, and defense; (III) carbohydrates; and (IV) nitrogen metabolism, which ranged from $53 \%$ to $64 \%$ of the total subsystems among the examined strains (Figure 3B).

In the Venn diagram based on the chromosome sequences, 3,378 gene clusters were identified, of which 2,604 were shared by all four genomes analyzed (Figure $3 \mathrm{C}$, top panel), 798 were orthologous, and 2,580 were characterized as single copies. The number of clusters identified in the four sequenced genomes ranged from 2,993 (CCRMRs304) to 3,064 (CCRMRsB7; Figure 3C, bottom panel). Only five clusters were strain-specific, four were specific to strain CCRMRsB7 (sequevar IIB-25), and one to strain CCRMRs287 (sequevar IIB-4); however, none of the clusters were associated with known functions. In the megaplasmid, 1,834 clusters were identified, of which 1,005 were shared by all four genomes analyzed (Figure 3D, top panel), 842 were orthologous, and 992 were identified as single copies. The number of clusters identified in the four genomes ranged between 1,308 (CCRMRsB7) and 1,627 (CCRMRs277; Figure 3D, bottom panel). Nine unique clusters were found; however, only five were associated with known functions, such as peptide transport, antibiotic biosynthesis, cholesterol metabolism (CCRMRsB7), transmembrane transport, and metabolic processes (CCRMRs287).

Among strains of the $R$. solanacearum Moko ecotype sequenced in this study, the genome of CCRMRsB7 is the largest sequenced to date, whereas that of CCRMRs287 is the smallest. However, both genomes are characterized by significant rearrangement of the $\mathrm{LCB}$, which requires further in-depth investigation. Moreover, the replicons of both these strains contain unique clusters. Our results indicate that the genomes of strains CCRMRsB7 (sequevar IIB-25) and CCRMRs287 (sequevar IIB-4) have distinct characteristics compared with other sequenced genomes examined thus far, and accordingly, both strains warrant further analysis in 


\section{Chromosome}

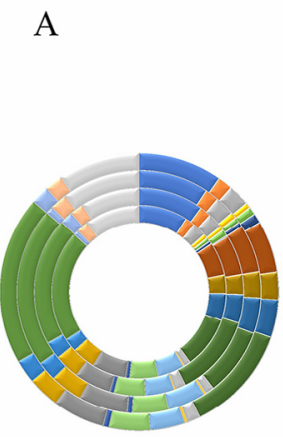

Subsystem Feature Counts (277/287/304/B7)*

Amino Acids and Derivatives (280/305/280/313)

Carbohydrates (138/141/137/15

Protein Metabolism (131/198/119/199)

Cofactors, Vitamins, Prosthetic Groups,Pigments (122/155/122/155) - Respiration $(86 / 88 / 85 / 96)$

Membrane Transport(84/96/84/96)

4 Fatty Acids, Lipids, and Isoprenoids (72/75/64/76)

Nucleosides and Nucleotides (67/68/62/70)

$\triangle$ DNA Metabolism (66/69/66/76)

- Stress Response (59/60/57/59)

$\square$ RNA Metabolism (47/48/46/5)

$\checkmark$ Virulence, Disease and Defense (35/36/34/40)

aratabolism of Aromatic Compounds (34/42/34/43)

1 Phosphorus Metabolism (30/33/30/33)

- Cell Wall and Capsule (24/27/24/27)

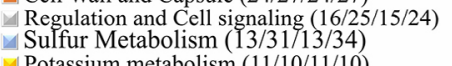

- Potassium metabolism $(11 / 10 / 11 / 10)$

Miscellaneous (10/16/10/15)

Phages, Prophages, Transposable elements, Plasmids (8/3/8/18)

Secondary Metabolism $(4 / 4 / 4 / 6)$

Dormancy and Sporulation $(1 / 1 / 1 / 1)$

Motility and Chemotaxis $(0 / 0 / 0 / 0)$

Cell Division and Cell Cycle $(0 / 0 / 0 / 0)$

Iron acquisition and metabolism $(0 / 0 / 0 / 0)$

Photosynthesis $(0 / 0 / 0 / 0)$
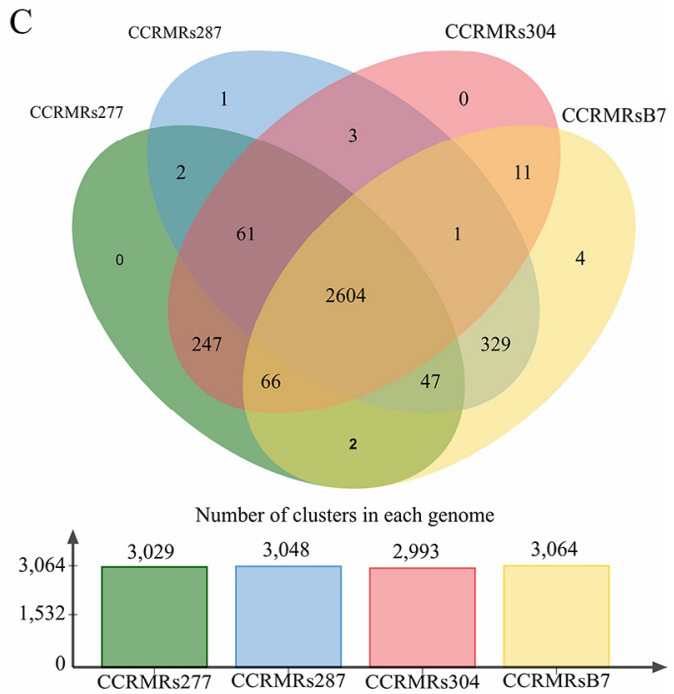

Megaplasmid
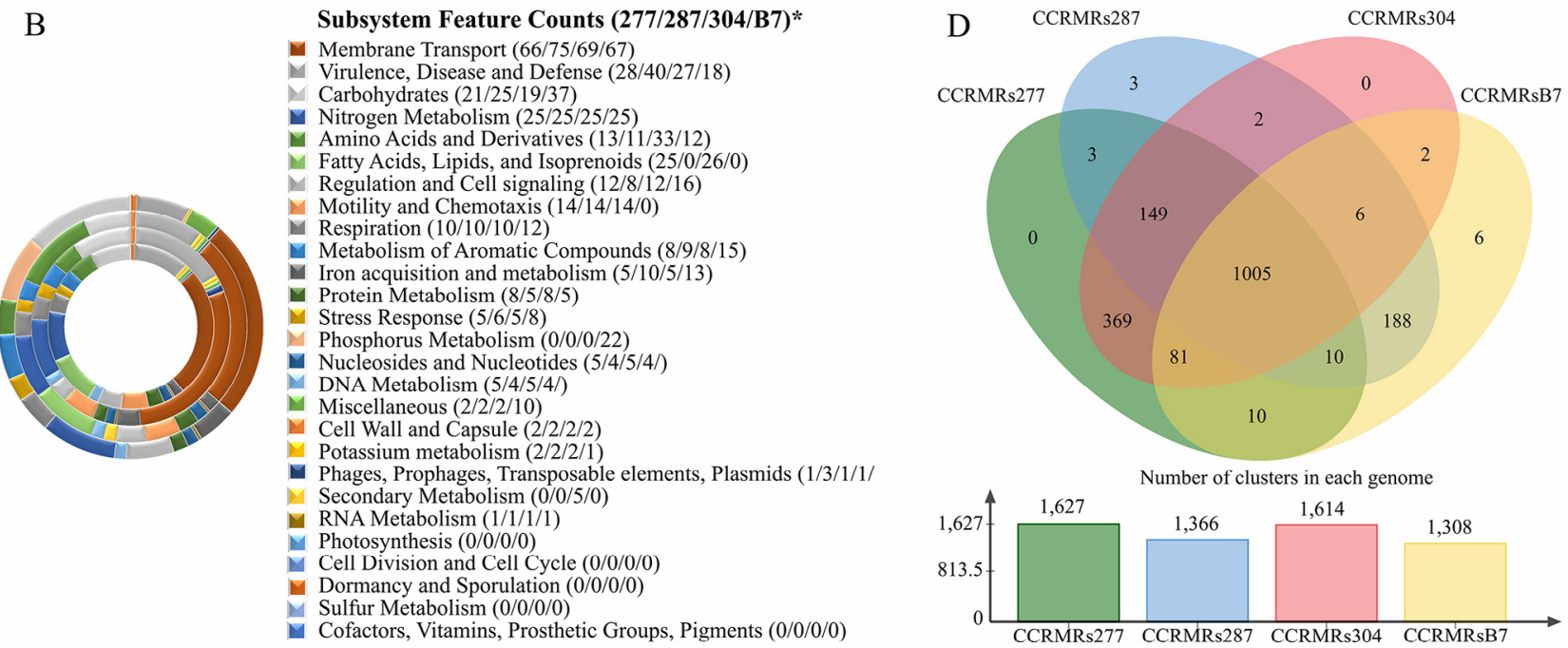

Figure 3 - Graphic representation of cluster subsystems of chromosomes (A) and megaplasmids (B) and Venn diagram of the chromosome (C) and megaplasmid (D) clusters of four strains of $R$. solanacearum belonging to the Moko ecotype $\left({ }^{*}\right.$ CCRMRs277 $=277$, sequevar IIA-24; CCRMRs287 $=$ 287, sequevar IIB-4; CCRMRs304 = 304, sequevar IIA-24; and CCRMRsB7 = B7, sequevar IIB-25) from Brazil, as well as the employed reference strains (UW163 and Po82, sequevar IIB-4).

this regard. Moreover, the genomic data elucidated by our study provides a theoretical basis that will facilitate the future identification, characterization, and phylogenetic analysis of the $R$. solanacearum Moko ecotype.

\section{Genome sequence accession number}

The accession numbers are as follows: GCA_014210395.1 for CCRMRs277, GCA_014210375.1 for CCRMRs287, GCA_014210335.1 for CCRMRs304 and GCA_014210345.1 for CCRMRsB7.

\section{Acknowledgment}

To the Conselho Nacional de Desenvolvimento Científico e Tecnológico - Brasil (CNPq) for the awarding a scholarship to Ana Karolina Leite Pais (Processo CNPQ ${ }^{\circ}$ 143017/2016-0) and the Laboratory of Bioinformatics and
Evolutionary Biology (LABBE) of Universidade Federal de Pernambuco (UFPE) for the availability of the servers used in bioinformatics analysis.

\section{Conflict of Interest}

The authors declare that there is no conflict of interest that could be perceived as prejudicial to the impartiality of the reported research.

\section{Authors Contributions}

EBS, GMRA and AMFS conceived and designed the study, AKLP, LVSS, ARGF and WJSJ conducted the in silico analysis, AKLP, JRS, WJSJ, VQB and MASG analyzed the data, AKLP and GMRA wrote the manuscript with contributions from WJSJ, AMFS, MASG and EBS, all authors read and approved the final manuscript version. 


\section{References}

Albuquerque GMR, Santos LA, Felix KCS, Rollemberg CL, Silva AMF, Souza EB, Cellier G, Prior P and Mariano RLR (2014) Moko disease-causing strains of Ralstonia solanacearum from Brazil extend known diversity in paraphyletic phylotype II. Phytopathology 104:1175-1182.

Assefa S, Keane TM, Otto TD, Newbold C and Berriman M (2009) ABACAS: algorithm-based automatic contiguation of assembled sequences. Bioinformatics 25:1968-9.

Brettin T, Davis JJ, Disz T, Edwards RA, Gerdes S, Olsen GJ, Olson R, Overbeek R, Parrello B, Pusch GD et al., (2015) RASTtk: A modular and extensible implementation of the RAST algorithm for building custom annotation pipelines and annotating batches of genomes. Sci Rep 5:8365.

Cellier G and Prior P (2010) Deciphering phenotypic diversity of Ralstonia solanacearum strains pathogenic to potato. Phytopathology 100:1250-1261.

Ceccatto V M (2015) Variação gênica. In: Ceccatto V M (ed) Biologia molecular. 2nd edition. EdUECE, Fortaleza, pp 63-86.

Darling ACE, Mau B, Blattner FR, Perna NT (2004) Mauve: multiple alignment of conserved genomic sequence with rearrangements. Genome Res 14:1394-1403.

Fegan M and Prior P (2005) How complex is the "Ralstonia solanacearum species complex". In: Allen C, Prior P and Hayward AC (eds) Bacterial wilt disease and the Ralstonia solanacearum species complex. APS Press, Saint Paul, pp 449-461.

Genin S and Denny TP (2012) Pathogenomics of the Ralstonia solanacearum species complex. Annu Rev Phytopathol 50:67-89.

Grant JR and Stothard P (2008) The CGView Server: a comparative genomics tool for circular genomes. Nucleic Acids Res 36:w181-w184.

Guidot A, Prior P, Schoenfeld J, Carrere S, Genin S and Boucher C (2007) Genomic structure and phylogeny of the plant pathogen Ralstonia solanacearum inferred from gene distribution analysis. J Bacteriol 189:377-87.

Gurevich A, Saveliev V, Vyahhi N and Tesler G (2013) QUAST: Quality assessment tool for genome assemblies. Bioinformatics 29:1072-1075.

Joshi NA and Fass JN (2011) Sickle: A sliding-window, adaptive, quality-based trimming tool for FastQ files Version 1.33 [Software].

Kurtz S, Phillippy A, Delcher AL, Smoot Ml, Shumway M, Antonescu C and Salzberg SL (2004) Versatile and open software for comparing large genomes. Genome Biol 5:R12.

Prior P and Fegan M (2005) Recent developments in the phylogeny and classification of Ralstonia solanacearum. Acta Hortic 695:127-136.

Pritchard L, Glover RH, Humphris S, Elphinstone JG and Toth IK (2016) Genomics and taxonomy in diagnostics for food security: Soft-rotting enterobacterial plant pathogens. Anal Methods 8:12-24.
Richter M and Rossello-Mora R (2009) Shifting the genomic gold standard for the prokaryotic species definition. Proc Natl Acad Sci USA 106:19126-19131.

Safni I, Cleenwerck I, Vos P De, Fegan M, Sly L and Kappler U (2014) Polyphasic taxonomic revision of the Ralstonia solanacearum species complex: proposal to emend the descriptions of Ralstonia solanacearum and Ralstonia syzygii and reclassify current $R$. syzygii strains as Ralstonia syzygii subsp. syzygii subsp. nov., R. solanacearum phylotype IV strains as Ralstonia syzygii subsp. indonesiensis subsp. nov., banana blood disease bacterium strains as Ralstonia syzygii subsp. celebesensis subsp. nov. and $R$. solanacearum phylotype I and III strains as Ralstonia pseudosolanacearum sp. nov. Int J Syst Evol Microbiol 64:3087-3103.

Santiago TR, Lopes CA, Caetano-Anollés G and Mizubuti ESG (2017) Phylotype and sequevar variability of Ralstonia solanacearum in Brazil, an ancient centre of diversity of the pathogen. Plant Pathol 66:383-392.

Seppey M, Manni M and Zdobnov EM (2019) BUSCO: Assessing genome assembly and annotation completeness. In: Kollmar M (ed) Gene prediction. Methods in Molecular Biology. Humana, New York, pp 227-245.

Wang Y, Coleman-derr D, Chen G and Gu YQ (2015) OrthoVenn: a web server for genome wide comparison and annotation of orthologous clusters across multiple species. Nucleic Acids Res 43:78-84.

Wick RR, Judd LM, Gorrie CL and Holt KE (2017) Unicycler: Resolving bacterial genome assemblies from short and long sequencing reads. PLoS Comput Biol 13:1-22.

Wicker E, Grassrt L, Coranson-Beaudu R, Mian D, Guilbaud C, Fegan $\mathrm{M}$ and Prior P (2007) Ralstonia solanacearum strains from Martinique (French West Indies) exhibiting a new pathogenic potential. J Appl Environ Microbiol 73:6790-6801.

\section{Internet resources}

Andrews S (2010), FastQC - A quality control tool for high throughput sequence data, http://www.bioinformatics.babraham.ac.uk/ projects/fastqc/ (accessed 20 May 2019).

National Center for Biotechnology Information (NCBI), https:// www.ncbi.nlm.nih.gov/ (Maio 15, 2019).

Ministério da Agricultura, Pecuária e Abastecimento (MAPA), http:// www.in.gov.br/materia/-/asset_publisher/Kujrw0TZC2Mb/ content/id/43461167/do1-2018-10-02-instrucao-normativan-38-de-1-de-outubro-de-2018-43461024 (Junho 20, 2019).

Associate Editor: Ana Tereza R. Vasconcelos

License information: This is an open-access article distributed under the terms of the Creative Commons Attribution License (type CC-BY), which permits unrestricted use, distribution and reproduction in any medium, provided the original article is properly cited. 